\title{
Médiévales
}

Langues, Textes, Histoire

55 | automne 2008

Usages de la Bible

\section{À propos de la mappemonde d'Ebstorf}

\section{Patrick Gautier Dalché}

\section{OpenEdition \\ Journals}

Édition électronique

URL : http://journals.openedition.org/medievales/5499

DOI : 10.4000/medievales.5499

ISSN : 1777-5892

\section{Éditeur}

Presses universitaires de Vincennes

\section{Édition imprimée}

Date de publication : 20 décembre 2008

Pagination : 163-170

ISBN : 978-2-84292-221-4

ISSN : 0751-2708

Référence électronique

Patrick Gautier Dalché, «À propos de la mappemonde d'Ebstorf », Médiévales [En ligne], 55 | automne 2008, mis en ligne le 20 mars 2011, consulté le 25 avril 2019. URL : http://journals.openedition.org/ medievales/5499; DOI : 10.4000/medievales.5499

Ce document a été généré automatiquement le 25 avril 2019

Tous droits réservés 


\title{
À propos de la mappemonde d'Ebstorf
}

\author{
Patrick Gautier Dalché
}

1 La mappemonde du monastère féminin d'Ebstorf en Saxe, diversement datée dans le courant $d u$ XIII $^{\mathrm{e}}$ ou du XIV ${ }^{\mathrm{e}}$ siècle, n'est plus connue que par des fac-similés antérieurs à sa destruction lors de la seconde guerre mondiale ${ }^{1}$. Par ses dimensions (une trentaine de feuilles de parchemin cousues ensemble mesurant au total 3,58 x 3,56 m et couvrant $12,74 \mathrm{~m}^{2}$ ), c'était la plus grande et la plus riche des mappae mundi monumentales du Moyen Âge. Outre des textes écrits dans les parties extérieures, elle comporte un grand nombre de vignettes représentant cités, peuples, animaux, ainsi que des légendes souvent très développées: au total près de 2345 unités textuelles et figurées. L'une de ses caractéristiques les plus frappantes est la présence de la tête, de la main et des pieds du Christ qui embrasse l'ensemble de la terre, selon une formule attestée dès le XII ${ }^{\mathrm{e}}$ siècle dans les représentations cosmo- et cartographiques.

2 Ce monument a suscité des recherches nombreuses qui, jusqu'à présent, ne sont pas parvenues à donner une image claire de son auteur, de sa date, des fonctions qui lui étaient assignées et, plus généralement, de sa place dans les représentations médiévales de l'espace. L'ouvrage de Jürgen Wilke s'emploie à répondre à ces questions par une multitude d'approches et des comparaisons minutieuses avec d'autres objets cartographiques ou avec la forme, l'écriture et le contenu de manuscrits copiés en Allemagne du Nord.

Il faut souligner d'entrée de jeu la nouveauté de ce travail qui, pour la première fois, examine la mappemonde d'Ebstorf sans a priori et sans être frappé de sidération devant ce qui a parfois été considéré comme un exploit dont il conviendrait de dévoiler l'énigme - ce qui n'est peut-être pas la meilleure façon d'aborder un objet historique. Pour cela, l'auteur revendique l'emploi conjoint de plusieurs disciplines : paléographie, histoire de l'art, histoire littéraire et histoire de la cartographie médiévale. Il est très raisonnablement conduit à une telle prise de position méthodologique par l'examen scrupuleux d'une historiographie déjà séculaire où la fantaisie et l'imagination des chercheurs se sont, comme souvent, donné libre cours. Bien qu'il sacrifie au mythe des 
«hommes du Moyen Âge » (p.13), il souligne dans son introduction, avec une fermeté justifiée, que de telles représentations exprimaient la réalité du monde pour leurs créateurs et leurs utilisateurs, dans un mélange où géographie, foi, histoire, mythologie interagissaient sans les contradictions que les modernes y repèrent souvent en d'inconscients anachronismes.

4 En me dispensant de suivre exactement un plan parfois diffus, j'examinerai les points essentiels de sa démonstration en m'attachant, plutôt qu'aux détails de l'histoire régionale saxonne sur lesquels de plus compétents auront à s'exprimer, à la méthode et aux questions d'ordre général qui se posent sur la matérialité de cet objet et sur ses fonctions.

5 La fabrication de la mappemonde occupe une place importante : nouveauté appréciable et utile, car des conclusions argumentées ou du moins des hypothèses sur son commanditaire, sa date, son milieu d'apparition et sa fonction peuvent en être plus judicieusement tirées. La carte équivaut à un volume in folio d'environ 180 feuillets, l'écriture n'en couvrant que 80 et l'investissement artistique correspondant à un cycle de 170 images au maximum. Les frais (10 à 15 marcs d'argent, à comparer avec le revenu annuel bien plus important $d u$ monastère d'Ebstorf), considérables sans être exceptionnels, ne contraignent donc pas à en attribuer nécessairement la production à une cour princière (ou impériale) et conduisent plutôt à penser qu'elle émane d'un scriptorium monastique ou cathédral.

6 La réalisation technique est ensuite abordée : l'ordre suivi pour assembler les feuilles de parchemin avant l'écriture et la peinture effectuées dans un deuxième temps, le tout sur une table où l'ensemble était disposé et déroulé selon les besoins, et dans un espace suffisant. Sous cette même rubrique sont examinés les moyens intellectuels mis en œuvre pour donner du monde une image cohérente, donc les sources de la carte - ce qui peut encore être considéré comme relevant de l'«atelier» du cartographe. À ce propos, l'historiographie balance entre deux conclusions opposées: soit que l'on considère la carte comme la copie d'une modèle d'origine antique, soit qu'on la tienne pour une création originale réalisée pour l'essentiel à l'aide de notions d'origine livresque. La première a conduit certains historiens imaginatifs à affirmer qu'elle conserve des traces plus ou moins importantes de la carte de la porticus Vipsania érigée par Agrippa, le gendre d'Auguste - dont nous ne connaissons ni la forme, ni le contenu, ce qui devrait suffire à détourner l'historien des reconstitutions arbitraires. La seconde opinion semble plus judicieuse, encore qu'elle tende à exclure sans preuves la continuité d'une tradition cartographique tardo-antique pourtant bien attestée, notamment par les travaux de l'auteur de ces lignes. Avec bon sens, J. Wilke les confirme de façon générale (sans toutefois les utiliser) : il est impossible d'imaginer qu'un monastère d'Allemagne du Nord ait pu disposer de l'énorme bibliothèque nécessaire à la création de légendes nombreuses et variées. Les parallèles précis avec une autre grande mappemonde conservée en Angleterre à Hereford (XIII ${ }^{\mathrm{e}}$ siècle) démontrent à eux seuls que les deux témoins ont une source cartographique ultime commune, élaborée à l'aide d'auteurs de l'Antiquité tardive et du haut Moyen Âge. La localisation de tel ou tel lieu, éventuellement connue par un texte, a dû être effectuée sur la reproduction d'un canevas cartographique préexistant permettant de le situer par rapport à d'autres lieux. Identifier des modèles cartographiques particuliers, comme il est fait ici et là sans démonstration développée, est en revanche périlleux. Quoi qu'il en soit, un dernier problème, essentiel, est fortement souligné sans pouvoir être résolu : rien ne nous garantit que la mappa conservée ne soit 
pas la copie directe et plus ou moins complète d'une carte antérieure - comme cela a été récemment démontré pour celle de Hereford : un texte de la fin du XII ${ }^{e}$ siècle en fait une description exacte, ce qui suppose un modèle de cette date dont la mappemonde réalisée un siècle plus tard est une copie ${ }^{2}$.

7 Avec une propension à tirer des conclusions définitives de recherches particulières, l'historiographie a notablement hésité sur la date de la mappemonde d'Ebstorf, proposant diverses solutions entre la première moitié $d u$ XIII $^{\mathrm{e}}$ siècle et le milieu du suivant. La date haute est liée à une hypothèse scurrile selon laquelle Gervais de Tilbury, auteur des Otia imperialia rédigés entre 1209 et 1214 et dédiés à l'empereur Otton IV, en serait responsable. Par des comparaisons avec d'autres réalisations artistiques de l'espace lunebourgeois - curieusement introduites par un rappel de l'exposé théorique de Hugues de Saint-Victor sur la nature duplice, texte et image, des mappae mundi (p. 54-55) ,- J. Wilke s'emploie à situer la réalisation des images vers 1300 , sans toutefois pouvoir les attribuer à un atelier précis, en particulier au monastère d'Ebstorf dont les témoins de l'activité artistique sont peu nombreux. Par des contacts avec des cartes produites dans d'autres aires culturelles, elle attesterait en outre des influences anglaises et hispaniques. Mais ceci demanderait à être précisé ou nuancé. De nombreuses médiations nous échappent sans doute; et ces correspondances, ici fort rares, pourraient remonter à des modèles communs disparus. À partir de comparaisons approfondies avec des documents produits en Basse-Saxe, l'analyse de l'écriture aboutit au même résultat. La main qui a tracé les textes se retrouve dans un groupe durablement actif à Ebstorf entre circa 1280 et circa 1330. Elle a notamment transcrit une hymne mariale au dos d'une miniature de l'Annonciation datée de 1300-1350, ce qui correspond à l'époque où le culte de Marie se développait sous l'impulsion du prévôt Albert (1293-1307). Pour autant que l'on puisse en juger à l'aide des reproductions, ces conclusions sont convaincantes.

Dès lors se pose la question de l'auteur. Étant donné le durable succès acritique de l'attribution à Gervais de Tilbury, un fort long chapitre qui parfois s'éloigne notablement de l'objet précis du livre, ainsi qu'un appendice, sont consacrés à son origine anglaise, à sa jeunesse, à sa carrière au service de l'empereur welf - il fut maréchal d'Arles - et au contenu de son œuvre dans ses éventuels rapports avec la mappemonde. Ce n'est pas le lieu de rappeler ici les constructions forcées par lesquelles les Otia imperialia ont été identifiés comme une source essentielle de la mappa mundi. J. Wilke n'a pas de mal à démontrer leur inanité : aucune preuve, aucun argument plausible ne les soutiennent. Il y a là, toutefois, un point à préciser. L'un des arguments des tenants de la thèse gervasienne provient du fait que, dans un manuscrit autographe des Otia (Vat. lat. 933), Gervais renvoie à des sources textuelles diverses et à une "mappa mundi». Pour tous les commentateurs, dont J. Wilke, ces annotations attesteraient que Gervais a utilisé une carte. En réalité, «mappa mundi » désigne ici très normalement un texte - effet de la double signification bien connue de l'expression médiévale ${ }^{3}$. Lorsque il annonce, dans un passage du livre I, qu'il traitera plus loin de Babylone, il écrit « ut infra in mappa mundi, decisione secunda, duce deo trademus ${ }^{4} »$. Il est donc impropre de tirer de là qu'il a utilisé une carte pour élaborer les Otia - même si, par ailleurs, cela n'est pas exclu. De même, lorsqu'il conclut une énumération des provinces de l'Empire romain en soulignant qu'il a proposé (il emploie le passé) « in summa naturalem prouinciarum ordinem et situm per tres orbis partes distinctarum in emendatiore pictura $^{5}$ ", ce "tableau plus correct» désigne la liste des provinces elle-même qu'il considère comme plus juste que l'autre type de picturae : celui que le vulgus appelle « mappa mundi ». Dans ce dernier cas seulement, il 
s'agit de cartes, sur lesquelles il porte un jugement négatif à cause des fautes touchant la «locorum ueritas» qui les déparent, et parce que l'emploi de l'expression «mappa mundi » à leur sujet est critiquable : c'est selon lui un usage du vulgaire. Nous avons donc là, non pas l'indication que Gervais aurait utilisé une ou des cartes, mais une réflexion sur le statut respectif du texte et de la carte quant à l'exactitude de la représentation.

L'interprétation du sens et de la fonction de la mappa mundi fait l'objet de plusieurs chapitres disséminés dans l'ensemble du plan. Des développements fort intéressants sont consacrés à l'« Erfahrungshorizont " géographique dont elle témoigne. L'affirmation du texte inscrit dans la marge supérieure droite, selon lequel la mappa mundi « non paruam prestat legentibus utilitatem, uiantibus directionem rerumque uiarum gratissime speculationis dilectionem» est ici prise au sérieux, contrairement à la tendance de l'historiographie qui dénie aux mappae mundi toute fonction que nous appelons pratique, mais sans se poser la question de l'adéquation de notre notion moderne de la pratique aux réalités intellectuelles médiévales. J. Wilke cherche en effet à déceler les intérêts du cartographe en examinant la façon dont il dépasse parfois le contenu de ses sources en accentuant la représentation de certaines régions. Il note qu'il a dû utiliser une mappemonde anglaise du type de celle de Hereford (ou un modèle commun) et une carte du type Beatus pour la France - ce qui suscite les mêmes réserves que celles plus haut exprimées : c'est possible, certes ; mais ce n'est ni ne peut être solidement prouvé. Des parallèles s'observent avec les itinéraires de l'Antiquité tardive (Itinéraire d'Antonin, Table de Peutinger, Cosmographe de Ravenne), qui ont été mis en œuvre par un intermédiaire cartographique - conclusions déjà formulées à propos de la mappemonde de Hugues de Saint-Victor par l'auteur de ces lignes non cité sur ce sujet. Des informations orales ont dû enfin être ajoutées à ces sources figurées et textuelles, ce qui permet de cartographier certains itinéraires qui, par l'abondance relative des détails topographiques, apparaissent privilégiés : les liaisons vers Londres, Paris, l'Italie du Nord révèlent l'importance et l'empreinte mentale des régions de l'Occident les plus actives économiquement (cartes dans le t. II, p. 22-28).

10 La mappemonde porte une empreinte welfe importante : Brunswick et Lüneburg, les deux principaux sièges du duché, sont particulièrement mis en valeur par des miniatures, de même que les tombeaux des martyrs d'Ebstorf. L'étude approfondie de ces particularités est menée dans l'intention de confirmer la date déjà exprimée et de décider si l'objet a pu être conçu et réalisé à la cour à la suite d'une commande, comme cela a été proposé antérieurement. La documentation permet de conclure que la date la plus vraisemblable correspond au règne d'Albert $\mathrm{I}^{\mathrm{er}}$ (1298-1308) et que le cartographe participait du milieu de la cour, mais que cela ne dit rien sur une commande officielle. La présence des tombeaux, l'accent mis sur son patron saint Maurice pointent davantage vers le monastère d'Ebstorf, d'ailleurs étroitement lié à la maison ducale, ce qui rend cette discussion un peu artificielle.

11 Les deux derniers chapitres abordent la fonction et l'utilisation de la carte. Ils n'occupent qu'une cinquantaine de pages : cela évite au lecteur de lire les habituelles généralités sur la charge "symbolique» des mappae mundi - trait qui sera sans doute reproché à l'auteur. Au contraire, c'est le public visé qu'il cherche à définir. L'emploi du latin suppose une préparation de celui qui observe tout en excluant sans doute, à cette date, un public principalement noble ou curial; la présence, dans les textes adventices, d'invitations au lecteur à aller chercher, soit sur la carte même, soit dans des élaborations textuelles, des renseignements plus complets, révèle plusieurs niveaux de réception. Mais 
l'emploi de la deuxième personne du singulier n'implique pas, comme le juge l'auteur, que cela s'adresse à une personne déterminée, tel un élève précis du cartographe, ni que la carte ait été faite pour lui : en bonne grammaire, le «tu » désigne très normalement l'ensemble des lecteurs potentiels des légendes. L'absence des significations morales ou allégoriques qui caractérisent les bestiaires ou les lapidaires suppose qu'elles étaient connues - ou qu'elles devaient être données par après, devant la carte même, ce qui entraine que sa fonction était, d'abord, pédagogique. L'usage des mappae mundi à cet effet est bien attesté, notamment par l'exemple de Hugues de Saint-Victor décrivant la structure d'une mappa mundi lors des collations qui, vers 1130, avaient lieu au cloître parisien. Il est dommage que J. Wilke, poursuivant dans l'exploration de la tradition victorine, invoque le Liber exceptionum sans savoir que l'auteur en est Richard de SaintVictor, recoure à l'édition de la Patrologie latine sans utiliser l'édition moderne et enfin sans se rendre compte qu'il ne s'agit pas d'une description de carte. La partie géographique du Chronicon de Hugues non cité, qui est tirée de l'analyse d'une carte, aurait été sur ce thème beaucoup plus éclairante. De même, J. Wilke sous-entend sans preuves que Hugues aurait renoncé à faire une mappemonde, remplacée par la description écrite, comme Richard. C'est ne pas tenir compte de nombreux indices bien connus. En particulier, la mappemonde qui se trouve dans un manuscrit des Étymologies d'Isidore de Séville (Munich, Bayerische Staatsbibl., Clm 10068) contemporain de Hugues et copié en Ile-de-France montre des correspondances frappantes avec le texte hugonien : elle doit être interprétée comme un exemplaire réduit de la mappemonde réelle décrite par le maître parisien. Cette carte ici contradictoirement datée du XI ${ }^{\mathrm{e}}$ siècle (t. II, Abb. 1, p. 38, en suivant un catalogue d'exposition) et du XII siècle (t. I, p. 66, n. 40) ne fait pas l'objet d'une enquête dans cette direction.

Un outil pédagogique, donc; mais pour quel public? Les nonnes d'Ebstorf eurent une école attestée à partir de 1307. J. Wilke pense que le responsable de la réalisation de la carte est le prévôt Albert. Mais il ne décide pas s'il la composa pour un élève particulier (celui auquel les verbes à la deuxième personne s'adresseraient) ou pour les religieuses. La première hypothèse semble peu vraisemblable; elle exclut a priori celles-ci de l'élaboration de la mappemonde, on ne voit pas pour quelles raisons. La signification d'ensemble abordée dans le dernier chapitre permet de préciser le contenu de l'enseignement auquel elle devait servir. Le Christ et ses stigmates, l'alpha et l'oméga qui flanquent son nimbe, l'image de Jérusalem correspondant à la description de la Jérusalem céleste dans l'Apocalypse, celle du ressuscité, montrent que cet enseignement portait sur l'histoire du salut et son exégèse. La carte représente la création dans toute sa diversité, en l'associant de façon purement graphique à la résurrection et au jugement dernier. Elle est un résumé de l'histoire sacrée. J. Wilke établit un rapprochement éclairant avec un texte marginal qui met en rapport les jours de la création avec la liturgie dominicale. Un tel rapprochement a été opéré dans un milieu monastique, évidence qui semble échapper à l'auteur. On entrevoit ici l'une des fonctions les plus profondes de ce type de représentation. La carte monastique interagit constamment, dans la conscience de qui la contemple (j'emploie ce terme à dessein) avec la liturgie, de façon à montrer la permanente actualisation de l'histoire du salut, hic et nunc, pour les religieux bénédictins et, plus généralement, pour le chrétien. Il n'y a pas de raisons de douter que la mappemonde ait été conçue par et pour des religieuses. Cette piste qui met l'accent sur sa fonction spirituelle n'est qu'effleurée, et l'hypothèse d'un élève particulier du prévôt Albert semble bien formelle. 
Face aux nouveautés importantes qu'il renferme, les erreurs ou les manques de ce travail sont secondaires. Outre les réserves déjà formulées sur l'influence de cartes anglaises ou hispaniques, sur le vocabulaire de Gervais de Tilbury et sur l'examen insuffisant des documents victorins, je redresserai quelques affirmations d'ordre général émanées de ce qu'il est convenu d'appeler l'histoire de la cartographie médiévale: les grandes mappemondes ne sont pas des "cartes TO", désignation qui ne s'applique qu'à des diagrammes cartographiques spécifiques; toutes les cartes médiévales ne sont pas "orientées à l'est», etc. Certaines attributions émanant de la même tradition historiographique sont sans fondement, comme les "cartes de saint Jérôme » ou la " carte d'Henri de Mayence ». J'y insiste, car la répétition de telles formulations confère une réalité indue à ces fictions savantes. Les transcriptions n'ont pas toujours été faites avec le scrupule exigé : par exemple, la citation d'une légende (t. I, p. 42) comporte deux fautes par comparaison avec la reproduction correspondante (t. II, p. 11); la désignation d'une très intéressante carte des provinces franciscaines n'est pas: «fratrum mappa ordinis » (t. I, p. 69 et t. II, p. 30) mais, comme cela est visible sur la reproduction : « ista est mappa ordinis» (t. II, p. 29). La liste des sources de la carte (t. II, p. 20) mentionne quelques noms provenant de travaux de seconde main et placés là sans critique : pour d'évidentes raisons relevant de l'histoire des textes qu'il n'est pas besoin de rappeler ici, ni Pomponius Mela, ni Vibius Sequester, ni Ptolémée ne peuvent être comptés parmi les sources au sens strict de la mappemonde. Quant au schéma des sources cartographiques (t. II, p. 21), il comporte des flèches orientées établissant des relations improbables entre des réalités incertaines : qu'est-ce que la « römische Weltkarte » qui serait à l'origine d'une «englische Weltkarte»? Que signifie l'attribution à "Castorius» de la Tabula Peutingeriana, sous-produit d'une historiographie dépassée? Quelle est la réalité de la «Karte des Orosius»? En quoi les cartes du type Beatus en dépendent-elles? Indépendamment de ces affirmations sans preuves et de ces attributions fantaisistes, documenter de telles relations controuvées postulées par l'histoire de la cartographie médiévale demanderait des démonstrations qui ne sont pas faites - et qui d'ailleurs sont impossibles.

Ce travail parfaitement présenté, qui fournit dans le deuxième volume un abondant et la plupart du temps judicieux appareil de schémas, de transcriptions et de reproductions, est appréciable par l'accent mis sur les caractéristiques matérielles de la mappemonde de façon à en éclairer le sens et la fonction. Quant à la date, à l'auteur et à ses buts, il apporte de nombreux éléments habilement critiqués. En mettant l'accent sur la réalité, à l'époque de sa réalisation, de l'espace tel qu'il est représenté pour ceux qui contemplaient la carte, il se donne les moyens de pénétrer dans la conscience de son auteur et de ses utilisateurs bien plus profondément que ceux qui se plaisent aux généralités sur la "pensée médiévale» et sur ses différences tout aussi générales avec les fondements de la cartographie moderne. De telles approches, dont l'ouvrage est un témoin valeureux, doivent être encouragées, si du moins l'on souhaite acquérir une meilleure compréhension des mappae mundi. 


\section{NOTES}

1. J. WILKE, Die Ebstorfer Weltkarte, Bielefeld, Verlag für Regionalgeschichte, 2001 ,Veröffentlichungen des Instituts für historische Landesforschung der Universität Göttingen, Band 39, 2 vols (I : Textband ; II : Tafelband), 347 et 167 p., une reproduction en dépliant.

2. Cf. S. WESTREM, The Hereford Map, Turnhout, 2001 (Terrarum orbis, 1).

3. Cf. P. GAUTIER DALCHÉ, « Les sens de mappa (mundi) (IV $-\mathrm{XIV}^{\mathrm{e}}$ siècle) », Archivum latinitatis medii aevi, t. 62, 2004, p. 187-202.

4. I, 20, éd. S. E. BANKS, J. W. BINNS, Gervase of Tilbury Otia imperialia. Recreation for an Emperor, Oxford, 2002 (Oxford medieval texts), p. 116.

5. II, 25 (ibid., p. 526).

\section{AUTEUR}

\section{PATRICK GAUTIER DALCHÉ}

EPHE, IV ${ }^{\mathrm{e}}$ Section, 45-47 rue des Écoles, F-75005 Paris 